\title{
Les convertases des prohormones et le système nerveux
}

Les convertases sont impliquées dans le processus de maturation des neuropeptides formés à partir de précurseurs polypeptidiques. Ces enzymes de clivage agissent de façon spécifique par la reconnaisance d'un site constitué, le plus souvent, de deux acides aminés basiques (lysine et arginine). Récemment, deux convertases PC1 et PC2 ont été identifiées. Celles-ci sont exprimées dans les neurones de nombreuses régions des systèmes nerveux central et périphérique. Les convertases PC1 et PC2 clivent les produits des gènes codant pour la proopiomélanocortine (POMC) et la lutéolibérine (LH-RH) respectivement. L'expression qualitative et quantitative d'une grande variété de polypeptides actifs est soumise à la régulation des convertases.

Mieczyslaw

Marcinkiewicz Nabil G. Seidah Michel Chrétien

\section{ADRESSE}

M. Marcinkiewicz : Chercheur senior. Laboratoire de Neuroendocrinologie Moléculaire. N.G. Seidah : Directeur, Laboratoire de Biochimie Neuroendocrinienne, Professeur titulaire, Université de Montréal. M. Chrétien : Directeur. Laboratoire de Neuroendocrinologie Moléculaire. Université de Montréal. Institut de Recherches Cliniques de Montréal (affilié à l'Université de Montréal et à l'hôpital Hôtel-Dieu de Montréal), 110, avenue des Pins-Ouest, Montréal (Québec) H2W 1 R7

\section{Introduction}

Le phénomène de maturation protéolytique est particulièrement important dans les systèmes nerveux et endocrinien, dans la mesure où la grande majorité des peptides actifs, à l'exception du glutathion et de la carnosine, proviennent du clivage d'un précurseur par protéolyse limitée et contrôlée. Celle-ci s'effectue par les enzymes de maturation qui agissent au niveau de sites spécifiques, le plus souvent composés de deux acides aminés basiques (lysine et arginine). Ce site est constitué dans quelques rares cas de résidus mono et multibasiques (figure 1) [1, 2].

Cependant, tous les sites potentiels d'un précurseur donné ne sont pas reconnus par les convertases et c'est seulement un nombre limité d'entre eux qui va permettre le clivage du précurseur. Ce mécanisme de pro- téolyse différentielle est de plus fonction du contexte cellulaire. L'exemple du précurseur de la proopiomélanocortine (POMC), qui contient 8 sites potentiels de clivage protéolytique illustre un tel mécanisme. La POMC qui est exprimée dans l'hypophyse va être clivée à des sites différents et donc produire des peptides différents au niveau du lobe antérieur et intermédiaire de l'hypophyse (figure 2). C'est ainsi qu'au niveau du lobe antérieur dans les cellules corticotropes (figure 2), seuls 4 des 8 sites vont être utilisés, aboutissant à la formation du peptide $\mathrm{N}$-terminal, du peptide JP (joining peptide), de l'ACTH (corticotropine), de la $\beta$-LPH ( $\beta$-lipotropine) ainsi que de la $\gamma$-LPH et de la $\beta$-endorphine mais en quantité moindre. Au niveau du lobe intermédiaire (figure 2), dans les cellules mélanotropes, les produits de clivage sont constitués par les 


\section{RÉFÉRENCES}

1. Chrétien M, Sikström R, Lazure C, et al. Expression of the diversity of neural and hormonal peptides via the cleavage of precursor molecules. In : Martinez J, ed. Peptide hormones as prohormones. England: Ellis Horwood Ltd, 1989 : 1-24.

2. Lazure C, Seidah NG, Pélaprat D, Chrétien M. Proteases and post-translational processing of prohormones : a review. Can $J$ Biochem Cell Biol 1983 ; 61 : 501-15.

3. Fricker LD, Adelman JP, Douglass J, Thompson RC, Von SR, Hutton J. Isolation and sequence analysis of cDNA for rat carboxypeptidase E [EC3. 4. 17. 10] a neuropeptide processing enzyme. Mol Endocrinol $1989 ; 3$ : 666-73.

4. Eipper BA, Park LP, Dickerson IM, et al. Structure of the precursor to an enzyme mediating $\mathrm{COOH}$-terminal amidation in peptide biosynthesis. Mol Endocrinol 1987 ; 1: 777-90.

5. Fuller RS, Brake A, Thorner J. Yeast prohormone processing enzyme (KEX2) (gene product) is a $\mathrm{Ca}^{++}$-dependent serine protease. Proc Nall Acad Sci USA 1989 ; 86 : 1434-8.

6. Roebroek AJM, Schalken JA, Lcunissen JAM, Onnekink HPJ, Gloemers WJM, Van de Ven WJM. Evolutionary conserved close linkage of the $c$-fes/fps proto-oncogene and genetic sequences encoding a receptorlike protein. $E M B O J 1989 ; 5$ : 2197-202.

7. Van Den Ouweland AMW, Van Duijnhoven HLP, Keizer GD, Dorssers LCJ, Van de Ven WJM. Structural homology between the human fur gene product and the subtilisin-like protease encoded by yeast KEX2. Nucleic Acids Res 1990；18: 664.

8. Seidah NG, Gaspar L, Mion P, Marcinkiewicz M, Mbikay M, Chrétien M. cDNA sequence of two distinct pituitary proteins homologous to Kex2 and Furin gene products : tissue-specific mRNAs encoding candidates for pro-hormone processing proteinases. DNA Cell Biol 1990; 6 : 415-24

9. Smeekens SP, Avruch AS, LaMendola J, Chan SJ, Steiner DF. Identification of cDNA encoding a second putative prohormone convertase related to PC2 in AtT20 cells and islets of Langerhans. Proc Natl Acad Sci USA 1991; 88 : 340-4

10. Smeekens SP, Steiner DF. Identification of a human insulinoma cDNA encoding a novel mammalian protein structurally related to the yeast dibasic processing protease Kex2. J Biol Chem 1990 ; 265 : 2997-3000.

11. Seidah NG, Marcinkiewicz M, Benjannet $S$, et al. Cloning and primary sequence of a mouse candidate prohormone convertase PC1 homologous to PC2, Furin and Kex2 : distinct chromosomal localization and messenger RNA distribution in brain and pituitary compared to PC2. Mol Endocrinol $1991 ; 5: 111-22$. mêmes peptides qui sont décrits dans les cellules corticotropes et également de peptides additionnels tels que l' $\alpha$ MSH ( $\alpha$-mélanotropine), le CLIP et la $\beta$-MSH, issus d'une maturation plus extensive de la POMC. Par ailleurs, au niveau de l'hypothalamus dans le noyau arqué, le mécanisme de la maturation de la POMC sem- ble similaire à celui observé dans le lobe intermédiaire de l'hypophyse.

Il est à noter que le clivage des prohormones par les convertases expose dans la région C-terminale un ou plusieurs acides aminés basiques. L'excision de ces dernières s'effectuc par l'action de la carboxypeptidase E (CPE) [3]. Cette réaction peut être

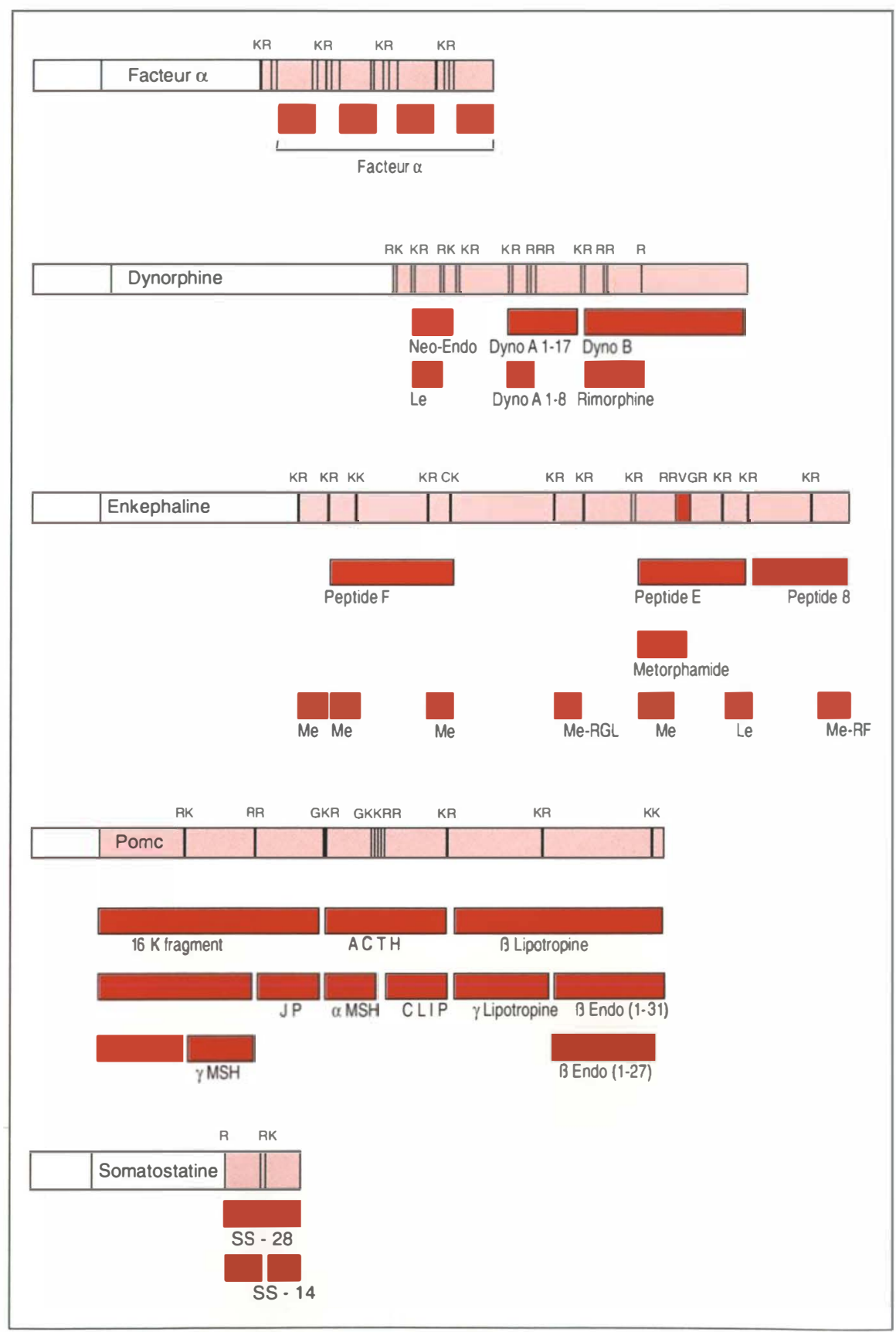

Figure 1. Exemple de précurseurs hormonaux et peptidiques. Les sites de clivage sont indiqués par les lettres $K$ (Lys) et $R$ (Arg). 
suivie d'une amidation de la région C-terminale par l'enzyme d'amidation (PAM) [4] ct/ou d'une acétylation de l'acide aminé $\mathrm{N}$-terminal par l'intermédiaire d'une acétyltransférase non encore caractérisée.

\section{Découverte des convertases}

Le phénomène de maturation protéolytique des pro-protéines est connu depuis plus de vingt-cing ans et ce n'est que récemment que les enzymes impliquées dans ce processus ont été identifiées. C'est par la découverte de l'enzyme de maturation du facteur d'accouplement de la levure Kex2 (ykexin) (figure 3) [5], que la recherche de l'identification des convertases des eucaryotes prit de l'ampleur. Le facteur d'accouplement ( $\alpha$ $\mathrm{MF}$ ) (figure 1) est synthétisé sous la forme d'un long précurseur contenant quatre copies identiques inactives de la protéine. Celles-ci sont libérées par l'action de la Kex2 au niveau des acides aminés Lys-Arg (figure 1). De fait, la Kex2 représente le prototype même des enzymes de maturation des prohormones et fait partic de la famille des protéinases à sérine du type subtilisine. Elle présente des homologies de séquences avec les différentes subtilisines et contient également le site catalytique composé de trois acides aminés (acide aspartique, histidine, serine). La séquence codant pour la région catalytique a été retrouvée dans le gène de la furine humaine (hFurin) [6, 7].

C'est en se basant sur le concept de la conservation des structures ayant une fonction importante, et en supposant une homologie partielle avec Kex2, que les convertases de systèmes nerveux et endocrinien de mammifères, PC1 $[8,9]$ et PC2 $[10,11]$ ont pu être découvertes. L'approche expérimentale consistait à employer la technique d'amplification génique par polymérisation en chaîne (PCR), en utilisant comme amorces des oligonucléotides dégénérés, dont les séquences furent dérivées à partir des structures conservées encadrant les sites catalytiques de Kex2 et de la subtilisine.

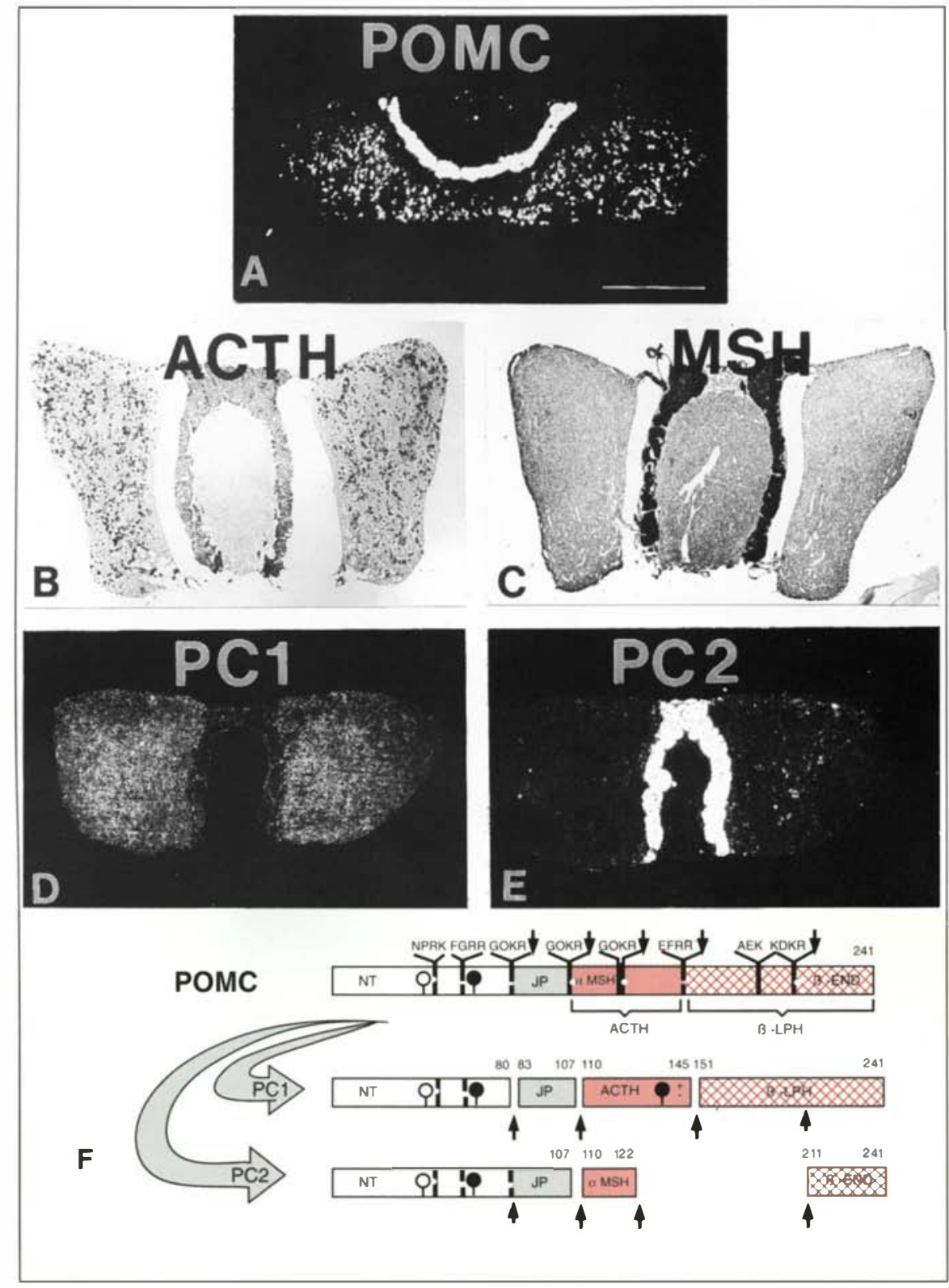

Figure 2. La maturation différentielle de la POMC dans la glande pituitaire de rat. (A) localisation de I'ARNm de POMC par hybridation in situ avec une sonde ARNc marquée au [35S]; (B) distribution immunocytochimique de I'ACTH et (C) de l' $\alpha$-MSH, visualisés par la méthode de peroxidaseantiperoxidase (PAP) à l'aide d'anticorps hautement spécifiques ; (D) distribution de I'ARNm de PC1 et (E) de I'ARNm de PC2 par hybridation in situ; (F) schéma de clivage de la POMC par PC1 et PC2. Abréviations : A - lobe antérieur ; 1 - lobe intermédiaire ; $P$ - lobe postérieur. Grossissement (A-E) $x$ $25 ;$ barre $=1 \mathrm{~mm}$.

\section{Les différents types de convertases}

La liste des convertases connues à ce jour et classées selon la longueur de la chaîne polypeptidique est présentée à la figure 3. La subtilisine bacté- rienne et Kex2 de la levure étant phylogénétiquement plus anciennes ont été souvent groupées sous l'appellation " subtilisine/Kex2 ". Le terme convertase a été étendu aussi à la furine de différentes espèces comme par exemple les furines humaines, 


\section{RÉFÉRENCES}

12. Roebroek AJM, Pauli IGL, Zhang Y, Van de Ven WJM. cDNA sequence of a drosophila melanogaster gene, Dfur1, encoding a protein structurally related to the subtilisin-like proprotein processing enzyme furin. FEBS Lett 1991; 289 : 133-7.

13. Kiefer MC, Tucker JE, Joh R, Landsberg KE, Saltman D, Barr PJ. Identification of a second human subtilisin-like protease gene in the fes/fps region of chromosome 15. DNA Cell Biol. 1991 ; 10 : 757-69.

14. Seidah NG, Day R, Hamelin J, Gaspar A, Collard MW, Chrétien M. Testicular expression of PC4 in the rat: Molecular diversity of a novel germ cell-specific Kex2/subtilisin-like pro-protein convertase Mol Endocrinol 1992 ; 6 : 1559-69.

15. Lusson J, Vieau D, Hamelin J, Day R, Barale JC, Chrétien M, Seidah NG. cDNA structure of mouse and rat PC5 : a novel candidate subtilisin/kexin-like pro-protein convertase expressed in endocrine and nonendocrine cells. Proc Natl Acad Si USA (sous presse).

16. Benjannet $S$, Rondeau $N$, Day $R$, Chrétien M, Seidah NG. PC1 and PC2 are proprotein convertases capable of cleaving proopiomelanoncortin at distinct pairs of basic residues. Proc Natl Acad Sci USA 1991; $88: 3564-8$

17. Wetsel WC, Seidah NG, and Collins S. Expression of candidate pro-LHRH processing enzymes in rat hypothalamus and immunomortalized hypothalmic neuronal cell hfurine, et de drosophile, dfurine [6, 12], à mPC1 (mouse pro-hormone convertase 1) et mPC2 (mouse prohormone convertase 2) de souris [8-11], à la PACE4 humaine [13] et à PC4 (pro-hormone convertase 4) [14] et PC5 (pro-hormone convertase 5) [15] (figure 3). Chez les mammiferes, nous connaissons actuellement la structure de 6 convertases. Ces enzymes peuvent être classées en groupes définis selon leur distribution tissulaire, ubiquitaire ou sélective. Les convertases furine et PACE4 sont ubiquitaires, PC1 et PC2 sont exprimées principalement dans les cellules endocrines et nerveuses. PC4 est synthétisée exclusivement dans les cellules germinales [14]. Le patron d'expression de PC5 n'est pas encore clairement défini.

La structure commune et essentielle à la fonction des convertases est représentée par la chaîne catalytique qui renferme un site actif composé de trois acides aminés (acide aspartique, histidine, serine) caractéristiques des subtilisines. Cette chaîne catalytique montre un degré élevé de conservation, alors que les séquences $\mathrm{N}$-terminales (le pro-segment) et C-terminales divergent davantage. L'identification des régions variables et constantes des convertases facilite la localisation de ces enzymes par les techniques de cytochimie d'hybridation (sondes cARN) ou d'immunocytochimie (anticorps), où les déterminants variables sont utilisés respectivement comme amorce et antigène spécifiques.

Récemment, il a été démontré dans le cas de la Kex2 et de la furine, que le pro-segment situé à l'extrémité $\mathrm{N}$ terminale est excisé par un mécanisme d'auto-clivage. Par analogie à la pro-subtilisine, la fonction de ce domaine $\mathrm{N}$-terminal assurerait le repliement de la structure tertiaire du zymogène. Toutes les convertases de mammiferes possèdent un ou plusieurs sites de glycosylation ainsi qu'une courte séquence Arg-Gly-Asp (RGD), potentiellement capable de reconnaître un ou plusieurs récepteurs de la famille des intégrines.

\section{Spécificité de l'activité enzymatique des convertases}

Le clonage des gènes codant pour les convertases PC1 et PC2 a permis d'étudier plus rigoureusement l'activité spécifique de ces enzymes. Ainsi, l'expression des ADNc de PC1 et PC2 dans différentes cellules en culture, en présence d'un substrat potentiel tel que la POMC, a pu être réalisée. Cette étude a été effectuée en utilisant le virus de la vaccine comme vecteur d'expression des ADNc spécifiques. Ces derniers sont introduits dans différentes lignées cellulaires telles que AtT-20, PC12 et BSC40, permettant ainsi l'expression sélective d'une enzyme avec un précurseur particulier. Cette étude a révélé que la POMC subit une fragmentation protéolytique distincte : le clivage de la POMC par PC1 aboutit à la formation de la corticotropine et de la $\beta$-lipotropine, alors que le clivage de la POMC par PC2 a pour résultat la production de la $\beta$ endorphine et l' $\alpha$-MSH (figure 2) [16]. C'est également la PC2 qui transforme le précurseur de la lutéolibérine en LH-RH active [17]. Les activités respectives des enzymes $\mathrm{PC} 1$ et $\mathrm{PC} 2$ semblent différentes dans le mécanisme de maturation de la proinsuline de rat exprimée dans les cellules Cos7. Alors que PC1 clive les deux jonctions Arg-Arg et Lys-Arg nécessaires pour libérer l'insuline I de rat, la PC2 clive uniquement le précurseur au niveau du site Lys-Arg, situé entre le peptide $\mathrm{C}$ et la chaîne A (figure 1) [18]. Il est intéressant de noter que dans la pro-insuline humaine ce site (Lys-Arg) résiste pourtant à l'action de PC1 [19]. Une telle résistance à $\mathrm{PC} 1$ pourrait s'expliquer par une différence dans la séquence au niveau du site de clivage P4 correspondant à la séquence RQKR chez le rat et LQKR chez l'homme [20]. Ainsi, la substitution de Arg par Leu à cette position pourrait altérer la reconnaissance de ce site par PC1. Il est donc possible que la maturation de la pro-insuline humaine nécessite l'action coordonnée de PC1 et PC2. Cependant, chez l'homme, l'activité concertée de PC1 et PC2 ne semble pas systématique. Par exemple, la pro-rénine humaine n'est activée que par PC1. Par ailleurs, cette activation s'effectue seulement dans les cellules de type endocrinien pourvues de granules sécrétoires, telles les somatomammotropes 
GH4 [21]. Cet exemple illustre à nouveau le clivage différentiel dans le processus de maturation des différents précurseurs selon le type de site de clivage et selon le contexte cellulaire.

\section{Les convertases \\ hypophysaires ; modèle pour le système nerveux}

Les données in vitro permettent d'analyser l'activité enzymatique d'une convertase donnée avec un substrat spécifique dans un contexte cellulaire particulier. Cependant il reste à démontrer in vivo la présence concomitante dans la même cellule de l'enzyme et du ou des substrats potentiels.

Des études cytochimiques poussées, visant à expliquer le phénomène de la maturation différentielle de la POMC en $\beta$-lipotropine ( $\beta$-LPH) et en ACTH dans les cellules corticotropes et en $\alpha$-MSH et $\beta$-endorphine dans les cellules mélanotropes (figure 2), ont été effectuées dans l'hypophyse en utilisant comme modèle animal celui de la souris [22]. L'ARN messager de PC1 est distri-

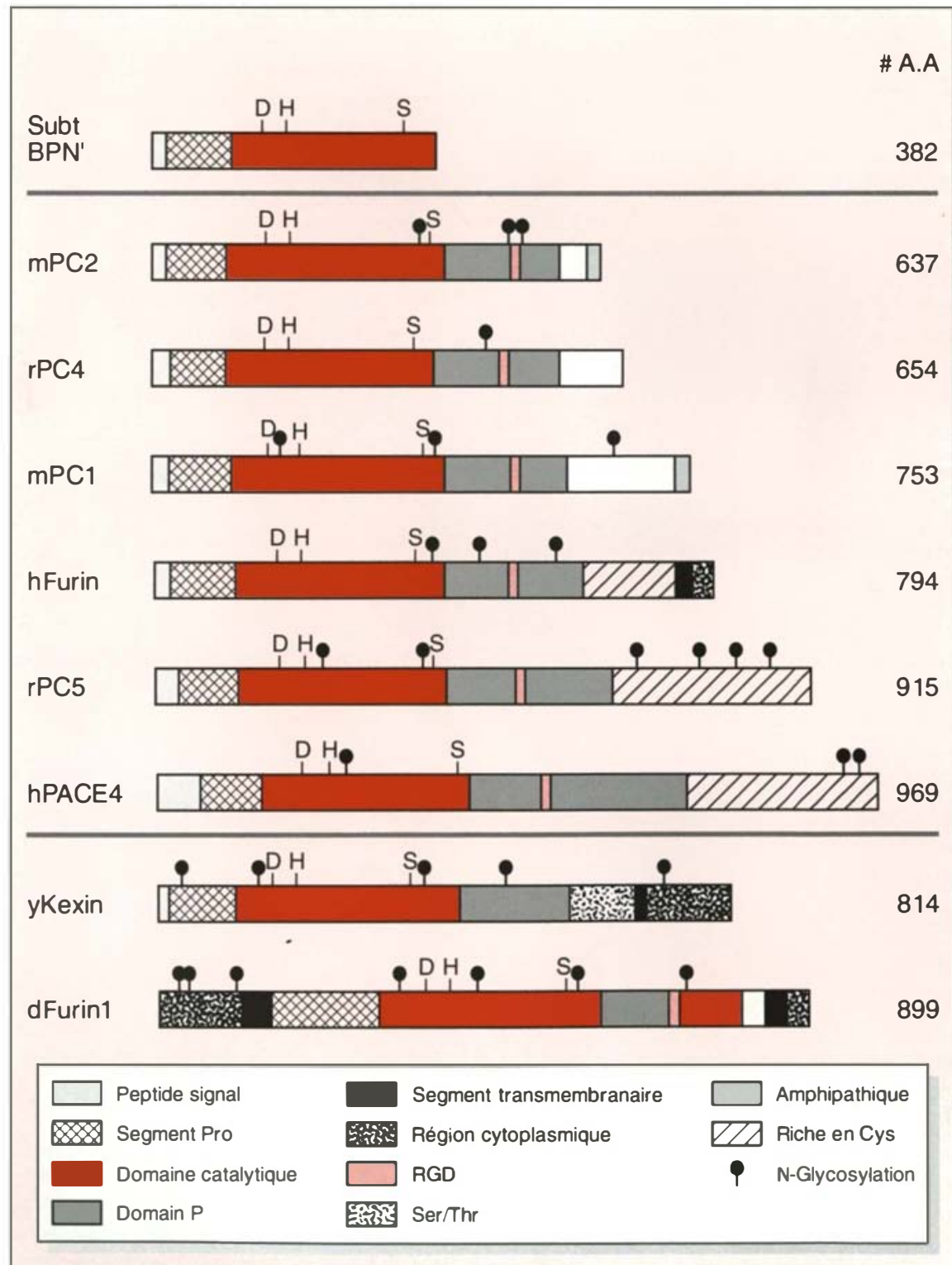

Figure 3. La famille des convertases : schémas de la structure. Le nombre d'acides aminés (AA) est indiqué à droite de la figure.

$\mathrm{m} / \mathrm{s} n^{\circ} 5$ vol. 9, mai 93 bué comme la POMC de façon équivalente dans les deux lobes de l'hypophyse, alors que celui de PC2 s'exprime surtout dans le lobe intermédiaire (figure 2). Il semble par conséquent pertinent de corréler l'activité préférentielle de l'enzyme PC2 dans le lobe intermédiaire avec la présence du produit de clivage $\alpha$-MSH également retrouvée préférentiellement dans ce lobe. A cette fin, nous avons étudié l'ontogenèse des cellules corticotropes incluant les cellules normales, atypiques et immatures chez la souris. Les résultats immunocytochimiques de double marquage ont montré qu'en effet : (i) PC1 est colocalisée avec l'ACTH, (ii) PC2 est colocalisée avec l' $\alpha$-MSH dans les limites de la période des deux premières semaines post-natale durant laquelle toutes les deux atteignent un pic d'expression maximum. En outre, cette colocalisation touche aussi quelques corticotropes atypiques chez la souris adulte [22]. Il est donc possible de postuler que c'est seulement lorsque le niveau d'expression de PC2 est suffisant que la maturation de la POMC s'exerce afin de produire l' $\alpha$-MSH et la $\beta$-endorphine. Le degré de plasticité de la maturation de POMC reflète donc son rapport avec la (les) convertase(s) endogène(s). Les niveaux de celles-ci peuvent varier en fonction des conditions physiologiques et développementales de la cellule. Dans certaines pathologies, comme dans les adénomes à des-acétyl $\alpha$-MSH - CLIP du syndrome de Cushing, le niveau des convertases peut être aussi modulé. Ainsi la variation de la production de la convertase endogène correspondrait parfaitement aux observations faites en physiologie et en pathologie quant à la production des peptides hormonaux.

\section{Localisation des PC1 et PC2 dans le système nerveux central et périphérique des rongeurs}

L'hybridation in situ a démontré l'expression de l'ARN messager de PC1 et de PC2 dans les neurones de multiples centres cérébraux, distribués depuis le lobe olfactif jusqu'à la moelle épinière, en passant par le 
cortex, les structures du système limbique telles que l'hippocampe et les amygdales, et dans l'hypothalamus, le thalamus, l'ćpithalamus et dans bien d'autres structures (figure 4) $[4,23$, 24].

De plus, les ćtudes immunocytochimiques (figure 5) semblent confirmer la localisation exclusive de PC1 et de PC2 dans les neurones, notamment dans les péricaryons des différents centres et dans les fibres nerveuses, tout spćcialement de l'ćminence médiane (figure 5D, D') [24]. Cette dernière structure, situce à l'intersection du système hypothalamohypophysaire, mérite une mention tout à fait particulière car cette structure renferme, à elle seule, plus d'une trentaine de neuropeptides dans les fibres de passage ou dans les terminaisons nerveuses du système porte [25]. Ainsi, les cartes cérébrales obtenues permettent de constater une ćtroite association de PC1 et de PC2 avec de nombreux neuropeptides, et peut-être aussi avec des membres de la famille des neurotropines, incluant le NGF (nerve growth factor), le BDNF (brain derived neurotrophic factor) et la NT-3 (neurotropine-3) [26].

La co-expression du NGF et de la convertase PC1 dans les ganglions spinaux

Pour devenir actives, les proneurotropines doivent subir un clivage au niveau d'un site Lys-XLys/Arg-Arg, sans doute par l'intervention d'une convertase. Cette hypothèse est renforcće par l'observation de la superposition des sites d'expression de PC1, PC2 et des différentes neurotropines dans les ganglions crâniens et spinaux du système nerveux périphérique. La figure 6 montre la localisation de l'AR Nm de $\mathrm{PC} 1$ (figure $6 \mathrm{~B}, D, H$ ) et $\mathrm{PC} 2$ (figure 6 $A, C, F$ et $J)$ dans les ganglions spinaux. Cette figure montre ćgalement des exemples de co-expression de PC1 et de PC2 avec le NGF (figure 6 $E, G, I)$ dans les neurones de ganglions spinaux, observée chez la souris nouveau-nće (figure $6 \mathrm{D}-G$ ) et la souris adulte (figure $6 \quad H-J$ ), c'est-à-dire avant et après myćlinisation. Bien qu'il existe une très bonne corrćlation entre l'ARNm du NGF et l'ARNm du PC1/PC2 au sein des ganglions chez l'adulte, le NGF s'exprime aussi dans les cellules de Schwann suite à une myćlinisation extensive. La présence de l'ARNm du NGF ne s'accompagne plus de celle de PC1 et PC2. Il est alors possible que, contrairement aux neurones, le NGF une fois synthétisć dans les cellules gliales de Schwann, subisse une maturation par une autre convertase, probablement la furine, dont la distribution tissulaire est plus génćrale [23]. La découverte de la colocalisation des ARN messagers des neurotropines avec PC1, PC2 et la furine ouvre un nouveau champ d'ćtudes sur les rôles que ces convertases peuvent exercer dans le développement, la physiologie et la pathologie du système nerveux central et périphérique.

\section{De quels paramètres dépend la maturation d'un précurseur : exemples de la pro-opiomélanocortine (POMC) et de la pro-rénine}

Quelques ćléments de réponse sont aujourd'hui disponibles.

1) Le type de site de clivage à un ou à plusieurs acides aminćs basiques joue, semble-t-il, un rôle dans la sćlection de la convertase compétente. L'exemple du site Lys-Lys-Arg-Arg nécessaire pour générer l' $\alpha$-MSH et du site Lys-Arg pour produire la $\beta$ endorphine, clivables par PC2 et non par PC1 (figure 2) [16] en est une bonne illustration.

2) La présence d'une ou de plusieurs enzymes pourrait être nćcessaire à l'efficacité de la maturation. Au cours de l'ontogenèse de l'hypophyse, la corrélation du niveau d'expression de PC2 et de la production d' $\alpha-\mathrm{MSH}$ apporte des arguments convaincants. Toute variation du niveau d'expression de la convertase dans la cellule (PC2 en l'occurrence) modifie le profil de maturation du précurseur (i.e. POMC) car celle-ci fluctue avec le niveau de PC2 (protéine) et de son ARNm [22]. Rappelons ćgalement l'action concertcee de PC1 et de PC2 dans le processus de maturation de la pro-insuline humaine [20].

3) La présence de granules de sćcrétion dans une cellule peut faciliter la maturation de certains précurseurs, la 

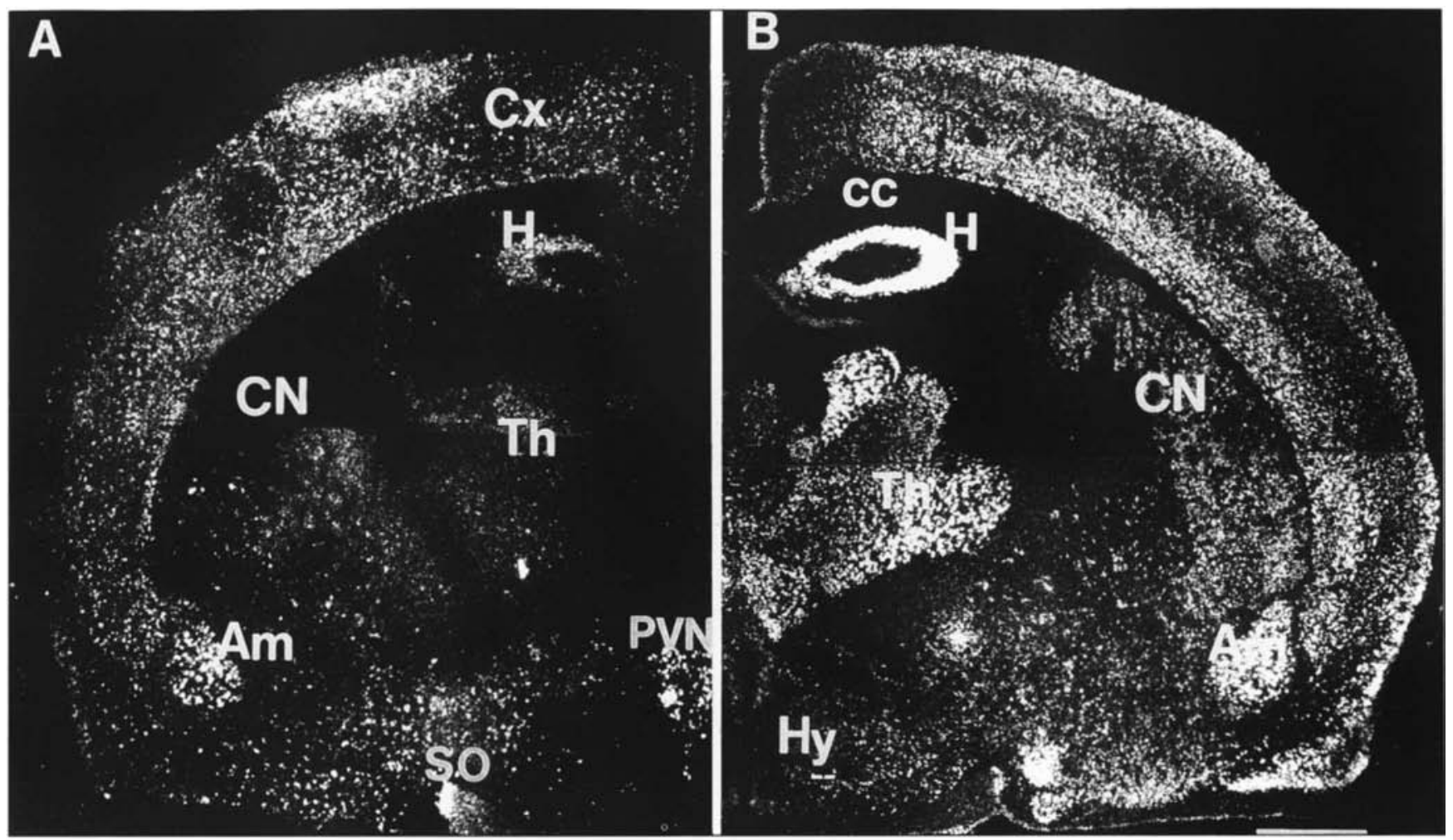

Figure 4. Expression de PC1 et PC2 dans le système nerveux central de souris. L'ARN messager de PC1 (A) et PC2 (B) est détecté par hybridation in situ dans le cortex (CX), I'hippocampe (H) et dans I'hypothalamus (Hy), cette dernière structure englobant les noyaux supraoptique (SO) et paraventriculaire (PVN). Dans les structures du thalamus (Th) et du noyau codé (CN) le signal d'hybridation de PC2 semble être prédominant par rapport à celui de PC1. Aucune hybridation n'est observée dans la masse de la substance blanche du corps calleux (cc). Grossissement $x$ 15; barre $=1 \mathrm{~mm}$.

pro-rénine humaine en est un exemple [21]. La présence des granules de sécrétion ne semble pas être déterminante pour la maturation de la $\mathrm{POMC}$ en ACTH, $\beta$-LPH et $\beta$ endorphine, mais est requise pour la production de l' $\alpha$-MSH [16].

4) La durée de la maturation intracellulaire est importante, car certains clivages se font précocement alors que d'autres surviennent tardivement. Cette conclusion est basée sur les études de la maturation de la POMC où il a été démontré qu'un clivage par PC2 en $\beta$-endorphine 1-31 (au niveau du lien Lys-Arg-) est précoce, alors que sa transformation par la même convertase en $\beta$-endorphine 1-27 (coupure après Lys-Lys) est beaucoup plus tardive [27].

\section{Conclusion}

L'étude des mécanismes de maturation des précurseurs polypeptidiques a permis d'identifier et d'analyser l'activité enzymatique des convertases. L'expression des convertases peut être ubiquitaire ou sélective. Par aillcurs un même précurscur contenant plusicurs sites potentiels de clivage peut subir une protéolyse différentielle. Celle-ci est fonction du nombre, de la nature des sites potenticls ainsi que du contexte cellulaire. D'autre part, l'efficacité de l'appareil sécrétoire ainsi que le temps nécessaire à la maturation semblent être des paramètres déterminants pour qu'une maturation correcte se produise. Les résultats obtenus, notam- ment avec les précurseurs tels que POMC, pro-rénine et plus récemment pro-LH-RH, permettent d'envisager une meillcure compréhension de l'activation des neuropeptides et des hormones peptidiques. La structure d'une partic des convertases est maintenant connue et l'analyse comparative des séquences révèle la présence de régions variables et constantes nécessaires à leurs activités spécifiques.

D'une manière générale, le cerveau ainsi que tous les systèmes composés de cellules excitables ont la capacité de produire une variété de peptides neuroactifs. Ceux-ci interviennent dans le théâtre de nombreux processus d'adaptation aux changements du milicu extéricur et dans le contrôle de 


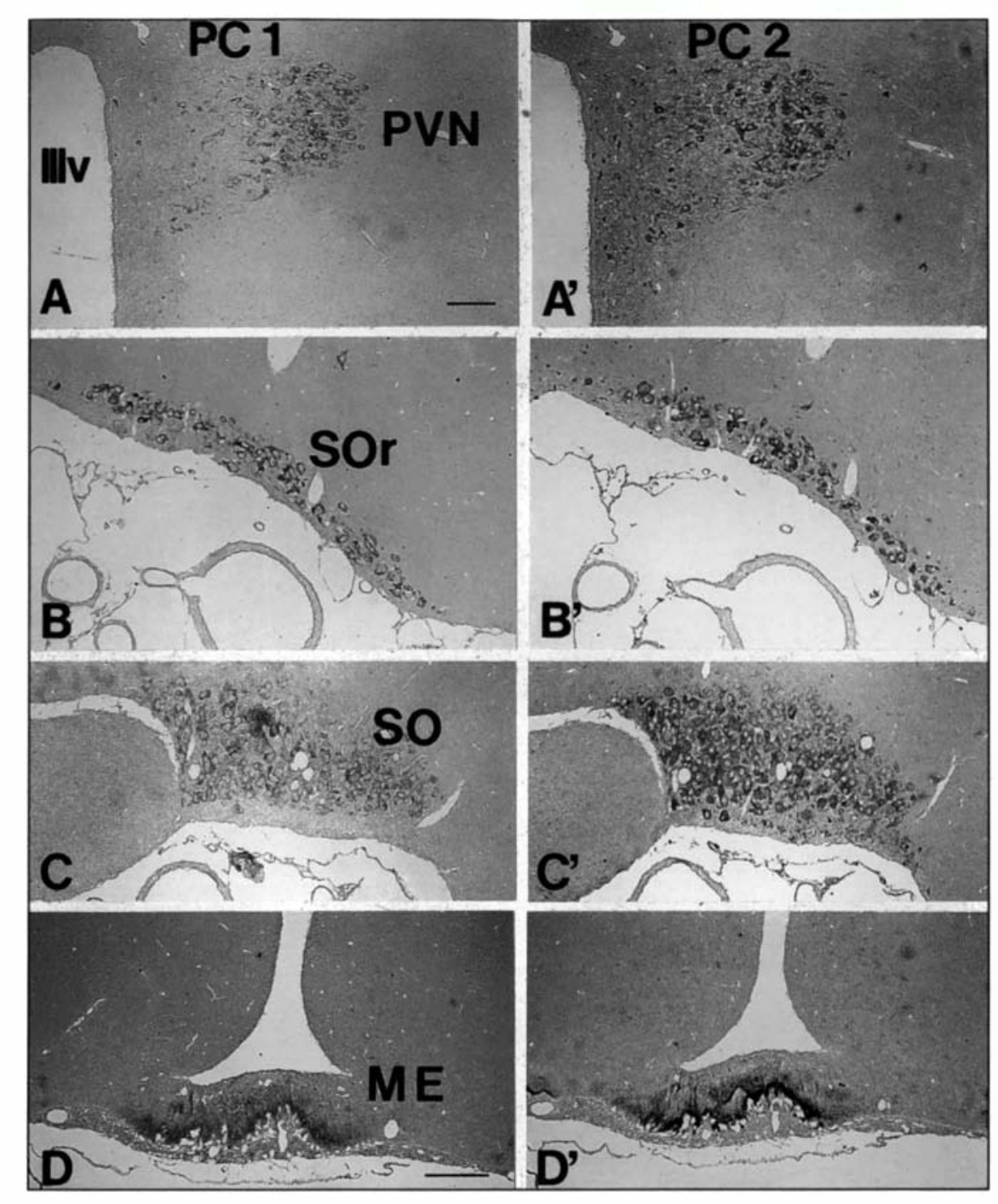

\section{RÉFÉRENCES}

22. Marcinkiewicz M, Day R, Seidah NG, Chrétien M. Ontogeny of the prohormone convertases PC1 and PC2 in the mouse hypophysis and their colocalization with ACTH and $\alpha-\mathrm{MSH}$. Proc Natl Acad Sci USA (sous presse)

23. Schäfer $M K-H$, Day $R$, Cullinan WE, Chrétien M, Seidah NG, Watson SJ. Gene expression of prohormone and proprotein convertases in the rat CNS : a comparative in situ hybridization analysis. $J$ Neurosci $1993 ; 13: 1258-79$

24. Marcinkiewicz M, Seidah NG, Chrétien M. Expression of mRNAs coding for the prohormone convertases PC1 and PC2 in the central and peripheral nervous system in adult and feta mouse. Society for Neuroscience, Annual Meeting Anaheim, Ca, October 1992, abstract \#121.15.

25. Palkovits ME. Neuropeptides in the Brain. In : Martini L, Ganong WF, eds. Frontiers in Neuroendocrinology. New York : Raven Press Ltd, 1988 : 1-44.

26. Barde YA. Throphic Factors and Neuronal survival. Neuron $1989 ; 2$ : 1525-34.

27. Zhou A, Bloomquist BT, Mains RE. The prohormone convertases PC1 and PC2 mediate distinct endoproteolytic cleavage in a strict temporal order during POMC biosynthetic processing. J Biol Chem (sous

Figure 5. Les convertases PC1 et PC2 dans I'hypothalamus du rat. Localisation immunocytochimique de $P C 1(A-D)$ et $P C 2\left(A^{\prime}-D^{\prime}\right)$ dans quelques centres peptidergiques de l'hypothalamus : $\left(A, A^{\prime}\right)$ le noyau paraventriculaire (PVN) ; $\left(B, B^{\prime}\right)$ le noyau supraoptique (SO) avec sa division rétrochiasmatique (SOr) $\left(C, C^{\prime}\right)$ et $\left(D, D^{\prime}\right)$ l'éminence médiane $(M E)$. Ces régions sont connues pour la production des différents neuropeptides, principalement la vasopressine et l'ocytocine. $I I I v=$ troisième ventricule. Grossissement $(A-C) \times 110 ;(D) \times 140$; barre $=100 \mu \mathrm{m}$.

l'homéostasie en assurant (i) la communication entre les neurones et leurs cibles, (ii) la différenciation et la croissance des tissus ainsi que (iii) le soutien et la régénération cellulaire. Tout semble indiquer que les converstases, y compris PC1, PC2, la furine et bien d'autres, jouent un rôle crucial dans cette voie, car, seules ou combinées, elles orchestrent d'une manière complexe la biosynthèse de cette variété de peptides bioactifs pour répondre aux besoins de plasticité et de développement du système nerveux.

Le rôle primordial des convertases dans tous les systèmes peptidergiques permet d'envisager assurément une meilleure compréhension de la physiopathologie dans de nombreuses maladies neurologiques 


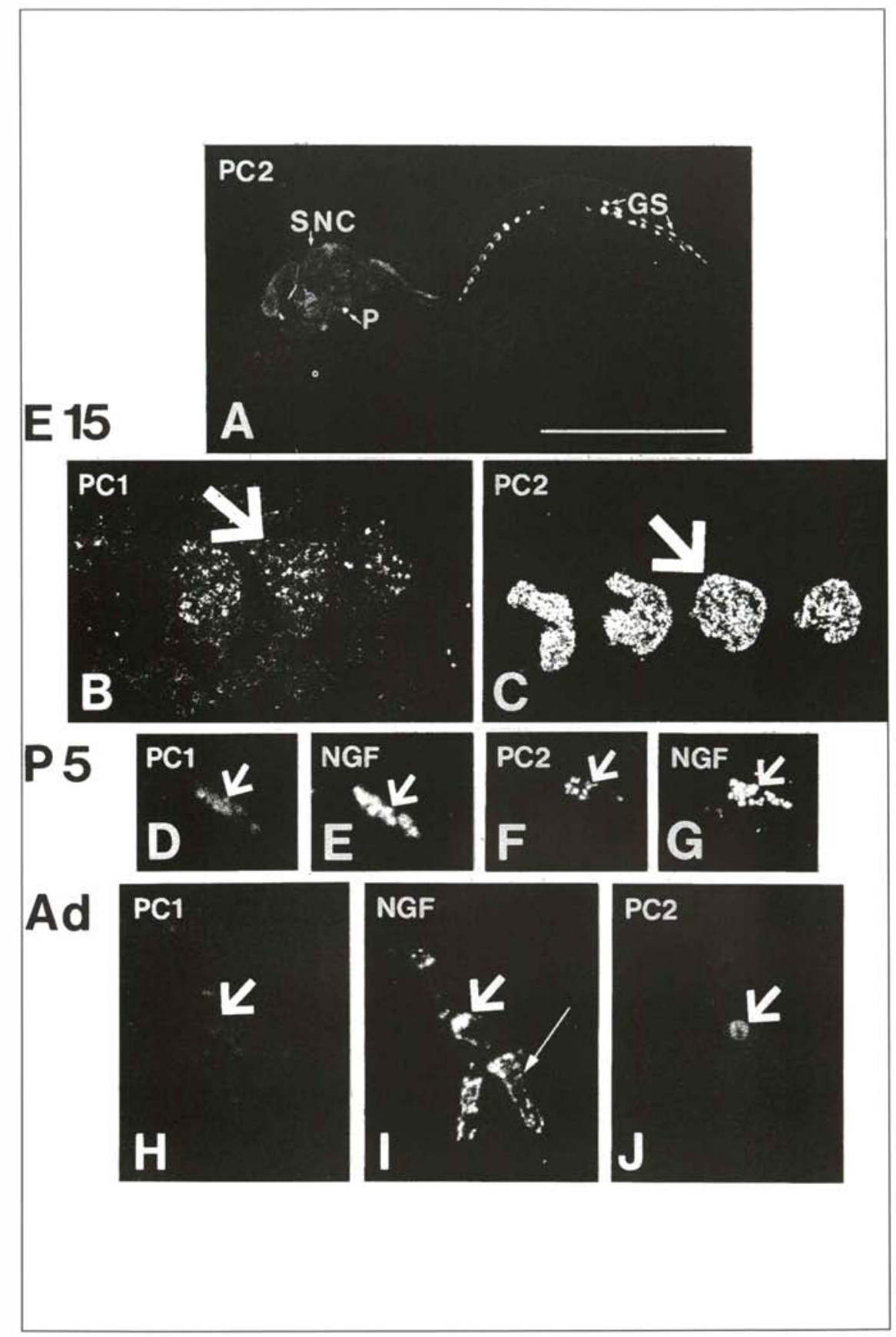

Figure 6. Les convertases PC1 et PC2 dans les ganglions spinaux de souris. Colocalisation avec le NGF.

(A) Une coupe sagittale de souris nouveau-née de 5 jours (P5) montre la localisation, par l'hybridation in situ, de l'ARNm de PC2 dans le système nerveux central (SNC), dans la glande pituitaire (P) et dans les ganglions spinaux (GS) du système nerveux périphérique.

(B) Localisation de l'ARNm de PC1 et (C) de PC2 dans les ganglions spinaux (GS) chez le fœtus 15 jours (E15) de la souris, à plus fort grossissement. $(D-G)$ La souris nouveau-née 5 jours (P5). L'ARNm de PC1 (D), PC2 (F) et NGF $(E$ et $G)$ est colocalisé dans les ganglions spinaux (flèches).

(H-J) La souris adulte. Une colocalisation de PC1 (H), PC2 (J) avec le NGF (I) dans les ganglions spinaux (grosse flèche). Pas de colocalisation dans les cellules de Schwann des nerfs périphériques, qui expriment I'ARNm du NGF (flèche fine).

Grossissement $(A, D-J) \times 4 ;(B$ et $C) \times 40$, barre $=1 \mathrm{~cm}$.

$\mathrm{m} / \mathrm{s} n^{\circ} 5$ vol. 9 , mai 93

\section{Summary}

Prohormone convertases and the nervous system

Active neuropeptides are produced through the processing of proproteins at pairs of basic amino acids (lysine and arginine). The enzymes responsible for the cleavage of pro-proteins (convertases) have been recently discovered and characterized. Enzymatic properties of two of them (PC1 and PC2) have been demonstrated based on the cleavage of proopiomelanocortin (POMC) and luteinizing hormone-releasing hormone (LH-RH), as model substrates. The mammalian convertases PC1 and PC2 were found to be expressed in neurons of the central and peripheral nervous systems. In these tissues PC1 and PC2 are likely responsible for the correct and sufficient production of a wide variety of tissue-specific neuropeptides and proteins. 